\title{
Evaluation of a Reflection Method on an Open-Ended Coaxial Line and its Use in Dielectric Measurements
}

\author{
R. Zajíček, J. Vrba, K. Novotný
}

This paper describes a method for determining the dielectric constant of a biological tissue. A suitable way to make a dielectric measurement that is nondestructive and noninvasive for the biological substance and broadband at the frequency range of the network analyzer is to use a reflection method on an open ended coaxial line. A coaxial probe in the frequency range of the network analyzer from $17 \mathrm{MHz}$ to 2 GHz is under investigation and also a calibration technique and the behavior of discrete elements in an equivalent circuit of an open ended coaxial line. Information about the magnitude and phase of the reflection coefficient on the interface between a biological tissue sample and a measurement probe is modeled with the aid of an electromagnetic field simulator. The numerical modeling is compared with real measurements, and a comparison is presented.

Keywords: complex permittivity, reflection method, coaxial probe.

\section{Notation and Units}

$\varepsilon \quad$ complex permittivity (-)

$\varepsilon_{0} \quad$ permittivity of free space, $\left(\varepsilon_{0}=8.854 \times 10^{-12} \mathrm{~F} \cdot \mathrm{m}^{-1}\right)$

$\varepsilon_{\mathrm{r}} \quad$ relative permittivity (-)

$\operatorname{tg} \delta \quad$ loss (dissipation) factor (-)

$\varepsilon_{\infty} \quad$ optical permittivity (-)

$\varepsilon_{\mathrm{s}} \quad$ static permittivity (-)

$\omega \quad$ angular frequency ( $\mathrm{rad}-1)$

$\tau \quad$ relaxation time (ps)

$f \quad$ frequency $(\mathrm{Hz})$

$j \quad$ imaginary unit $\left(\mathrm{j}^{2}=-1\right)$

$\alpha \quad$ distribution parameter (-)

$\sigma_{\mathrm{i}} \quad$ static ionic conductivity $\left(\mathrm{S} \cdot \mathrm{m}^{-1}\right)$

C capacitance (F)

$G \quad$ conductance $\left(\mathrm{S} \cdot \mathrm{m}^{-1}\right)$

S-parameter Scattering Parameter

$\mathrm{S}_{11} \quad$ Reflection Coefficient

MUT Material Under Test

FIT Finite Integration Technique

FFT Fast Fourier Transformation

TEM Transversal Electric and Magnetic Wave

\section{Introduction}

The dielectric properties of biological tissues are determining factors for the dissipation of electromagnetic energy in the human body and therefore they are important parameters in hyperthermia treatment, in microwave detection of tumors and in the assessment of exposure doses in basic research on interactions between electromagnetic fields and biological tissues [1].

Measurement of the dielectric parameters of biological tissue is a promising method in medical imaging and diagnostics. Knowledge of the complex permittivity in an area under treatment, i.e. knowledge of the complex permittivity of healthy and tumor tissues, is very important for example in diagnosing of tumor cell-nests in medical diagnostics or for engineers in the design of thermo-therapeutic applicators. Other interesting applications are 3D reconstruction methods for various biological tissues based on the layered uniform tissue model (skin, fat and muscle).

\section{Materials and method}

There are several measurement methods for measuring dielectric properties [2]. If we want to use a broadband measurement method that is nondestructive, noninvasive and can

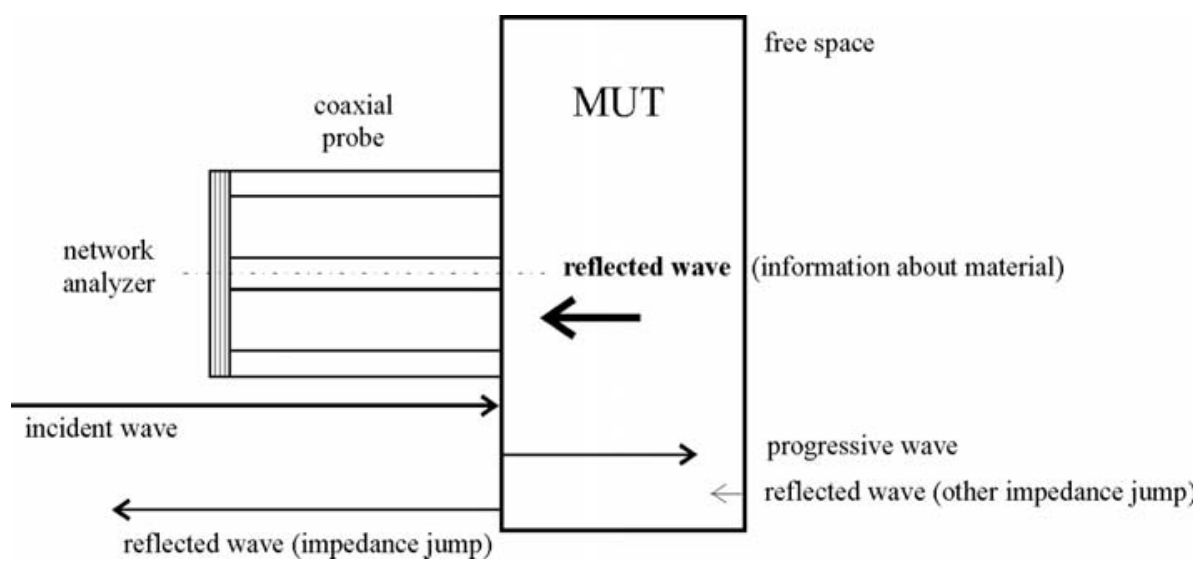

Fig. 1: Principle of the reflection method 


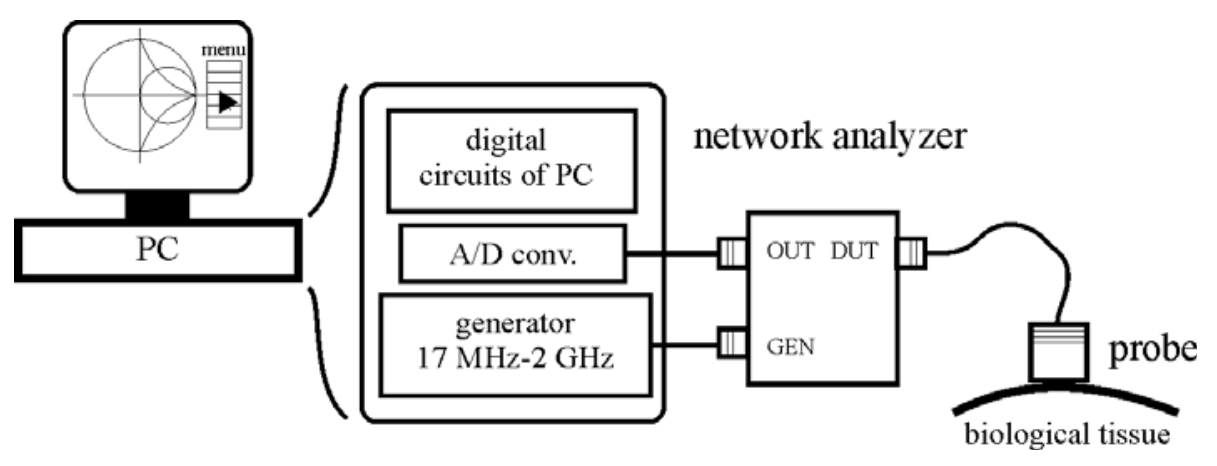

Fig. 2: Measurement system

offer possibilities for in vivo as well as in vitro measurements, we should choose a reflection method on an open-ended coaxial line.

The objective of the research reported here is to analyze an open-ended coaxial line sensor for in vivo and nondestructive measurements of complex permittivity, and to develop a precision measurement system. The interface between the measurement probe and the sample of biological tissue presents an impedance jump (Fig. 1). Biological tissue has extremely high permittivity values. At low frequencies, its relative permittivity is more than 100 and the value of the loss factor is more than 0.1. An exact evaluation is very difficult because the reflection coefficient $R$ is close to 1 . This means that only a very small part of the incident energy penetrates into the sample, and for this reason the obtainable information is very poor.

The reflection method on the open end of the coaxial line is a well-known method for determining these dielectric parameters [3]. This method is based on the fact that the reflection coefficient of an open ended coaxial line depends on the dielectric parameters of the material which is attached to it. To calculate the dielectric parameters from the measured reflection coefficient it is necessary to use an equivalent circuit of an open-ended coaxial line. To determine the values of the elements in this equivalent circuit we make a calibration using materials with known dielectric properties.

A typical measurement system using a coaxial probe method consists of a network or impedance analyzer, a coaxial probe, and software. Our measurements (Fig. 2) were done the aid of a sixport type network analyzer in the frequency range from $17 \mathrm{MHz}$ to $2 \mathrm{GHz}$.

\subsection{Dielectric theory}

The dielectric constant - relative permittivity $\varepsilon_{\mathrm{r}}-$ in our case describes the interaction of a biological tissue with an electric field, and because of the loss character of biological tissue it is a complex quantity.

$$
\frac{\varepsilon}{\varepsilon_{0}}=\varepsilon_{\mathrm{r}}=\varepsilon^{\prime}-j \varepsilon^{\prime \prime},
$$

where $\varepsilon_{\mathrm{r}}$ is relative permittivity and $\varepsilon_{0}$ is permittivity of free space.

The real part of the permittivity is a measure of how much energy from an external electric field is stored in a material. The imaginary part of the permittivity is a measure of how dissipative or lossy a material is to an external electric field.

The dissipation factor is defined by:

$$
\operatorname{tg} \delta=\frac{\varepsilon^{\prime \prime}}{\varepsilon^{\prime}} .
$$

It is important to note that complex permittivity is not constant; it changes with frequency, temperature, etc.

\subsection{Frequency dependence of complex permittivity}

The dielectric spectrum of a tissue is characterized by three main relaxation regions $\alpha, \beta$, and $\gamma$ at low, medium and high frequencies, and other minor dispersions such as the often reported $\delta$ dispersions. In its simplest form, each of these relaxation regions is the manifestation of a polarization mechanism characterized by a single time constant $\tau$ (relaxation time $\tau$ is a measure of the mobility of the molecules and dipoles that exist in a material), which to a first order approximation gives the following expression for complex relative permittivity $\varepsilon_{\mathrm{r}}$ as a function of angular frequency $\omega$.

$$
\varepsilon_{\mathrm{r}}=\varepsilon_{\mathrm{r}}^{\prime}-j \varepsilon_{\mathrm{r}}^{\prime \prime}=\varepsilon_{\infty}+\frac{\varepsilon_{\mathrm{s}}-\varepsilon_{\infty}}{1+j \omega \tau} .
$$

This is the well-known Debye expression, in which:

- $\varepsilon_{\infty}$ is the optical permittivity at high field frequencies (where $\omega \tau \gg 1$ ),

- $\varepsilon_{\mathrm{S}}$ is the static permittivity (at $\omega \tau \gg 1$ )

- and $j^{2}=-1$.

The dispersion magnitude is described as $\Delta \varepsilon=\varepsilon_{\mathrm{S}}-\varepsilon_{\infty}$.

However, the complexity of both the structure and the composition of biological material is such that each dispersion region may be broadened by multiple contributions to it. The broadening of the dispersion can be empirically accounted for by introducing a distribution parameter, thus giving an alternative to the Debye equation, known as the Cole-Cole equation

$$
\varepsilon=\varepsilon_{\infty}+\frac{\Delta \varepsilon}{1+(j \omega \tau)^{1-\alpha}},
$$

where the distribution parameter $\alpha$ is a measure of the broadening of the dispersion.

The spectrum of a tissue may therefore be more appropriately described in terms of multiple Cole-Cole dispersion

$$
\varepsilon=\varepsilon_{\infty}+\sum_{n} \frac{\Delta \varepsilon_{n}}{1+\left(j \omega \tau_{n}\right)^{1-\alpha_{n}}}+\frac{\sigma_{\mathrm{i}}}{j \omega \varepsilon_{0}},
$$

which, with a choice of parameters appropriate to each tissue, can be used to predict the dielectric behavior over the desired 
frequency range. $\sigma_{\mathrm{i}}$ is static ionic conductivity and $\varepsilon_{0}$ is the permittivity of free space.

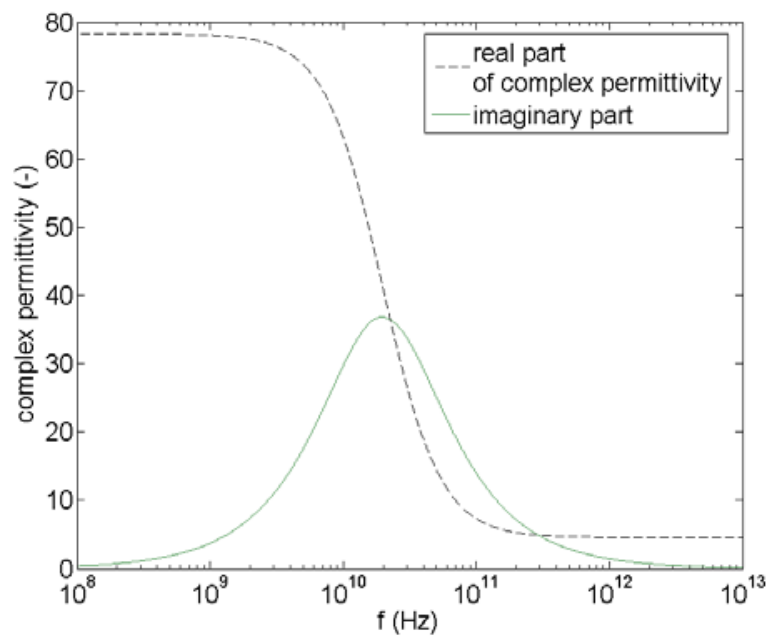

Fig. 3: Dielectric behaviour of distilled water at $30^{\circ} \mathrm{C}$

For distilled water, the dielectric constants are:

- $\varepsilon_{\infty}=78.3$,

- $\varepsilon_{\mathrm{S}}=4.6$,

- $\tau=8.07 \mathrm{ps}$,

- $\sigma_{\mathrm{i}}=2 \mathrm{~S} \cdot \mathrm{m}^{-1}$

- $\alpha=0.014$.

\subsection{Measurement probe}

A coaxial probe is created by an open end of a transmission line. The material can be measured by touching this probe to the flat face of a material and determining the reflection coefficient. For this measurement method we have developed a new type of coaxial measurement probe. This probe was created by adapting the standard $N$-connector
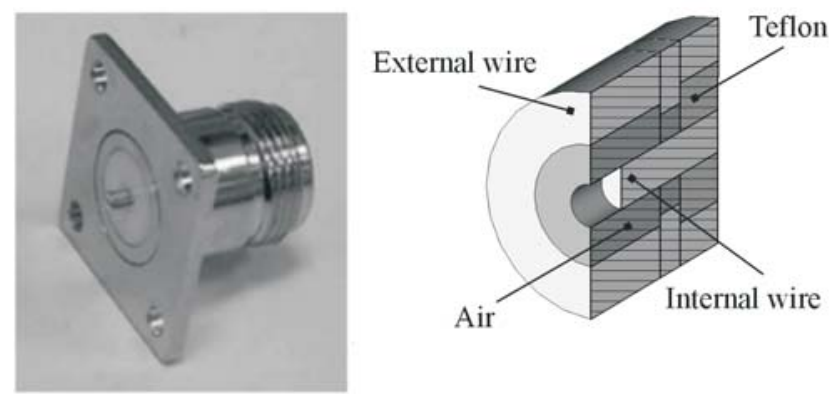

Fig. 4: N-connector and probe model

(Fig. 4), from which the parts for connecting to a panel were removed.

The reflected signal $S_{11}$ can be measured and related (Eq. 6) to the complex permittivity $\varepsilon_{\mathrm{r}}$ using an equivalent circuit of an open ended coaxial line.

$$
Y=j \omega \varepsilon_{\mathrm{r}} C+\sqrt{\varepsilon_{\mathrm{r}}^{5}} G .
$$

The equivalent circuit consists of two elements:

- $C$ is the capacitance between the internal and external wire out of the coaxial structure,

- $G$ is the conductance which represents the propagation losses.

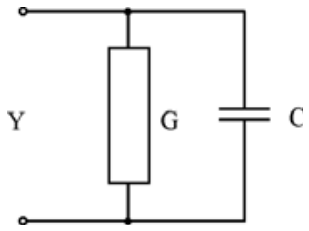

Fig. 5: Equivalent circuit of a measurement probe

The measurement probe radiates at higher frequencies (Fig. 6). So not only the fringing capacitance $C$ must be taken into account.
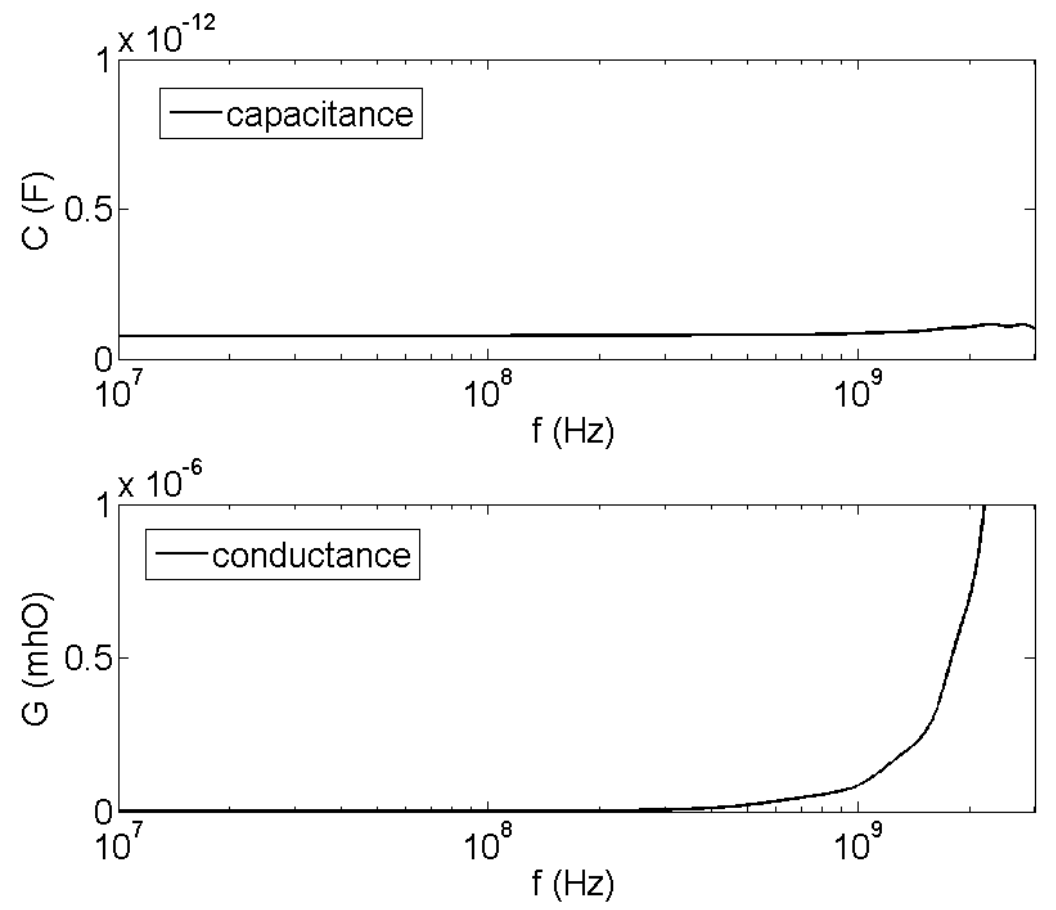

Fig. 6: Frequency behaviour of equivalent circuit elements 


\section{Numerical modeling}

A feasibility study of this measurement method involves numerical calculations and modeling (Fig. 7). The system that we modeled consisted of two parts, i.e. the sensor and the biological tissue. In order to model the $N$-connector, its dimensions were measured and the available catalogue data was studied. The biological tissue sample was modeled on the basis of available published data on relative permittivity $\varepsilon_{\mathrm{r}}$, loss factor $\operatorname{tg} \delta$ and conductance $\sigma$.

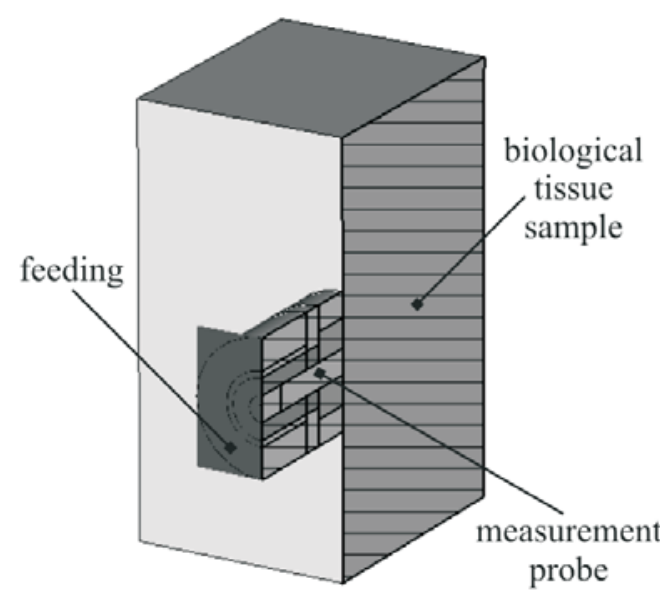

Fig. 7: Model of the measurement system

Numerical simulation based on the Finite Integration Technique (FIT) [5] is used to calculate the reflection coefficient on the interface between the coaxial probe and a sample of biological tissue. FIT is a discretization method which transforms Maxwell's equations in their integral form onto a dual cell grid complex, resulting in a set of discrete matrix equations. The structure is excited in the time domain with a Gaussian pulse. By performing the Fourier Transformation (FFT) and division we can obtain the response of the structure in the frequency domain. The properties and duration of this time signal are taken into account in order to obtain accurate and correct results. The waveguide port is used to feed the calculation with power and to absorb the returning power. For the waveguide port, the time signals and $S$-parameters $\left(S_{11}\right)$ are recorded during a solver run.

The numerical results will be compared with real measurements. Let us summarize the parametrs of the input simulator;

- the frequency range of the numerical calculation is given by the network analyzer frequency range from $17 \mathrm{MHz}$ to $2 \mathrm{GHz}$

- the wave port is used as a feeding element because of the coaxial structure of the probe and the transversal electric and magnetic (TEM) mode (wave) excitation

- the output of the simulation are the $S_{11}$-parameter (reflection coefficient) and a visualization of the magnitude of the electric field

- the model of the MUT-biological tissue is defined by dielectric parameters found in the literature (Table 1)
Table 1: Tabbed dielectric values of muscle biological tissue

\begin{tabular}{|r|c|c|c|}
\hline$f(\mathrm{MHz})$ & $\varepsilon_{\mathrm{r}}(-)$ & $\operatorname{tg} \delta(-)$ & $\sigma\left(\mathrm{S} \cdot \mathrm{m}^{-1}\right)$ \\
\hline 10 & 170.7 & 6.5 & 0.62 \\
\hline 20 & 110.6 & 5.2 & 0.64 \\
\hline 50 & 77.1 & 3.2 & 0.68 \\
\hline 70 & 70.8 & 2.5 & 0.69 \\
\hline 100 & 65.9 & 1.9 & 0.71 \\
\hline 200 & 60.2 & 1.1 & 0.74 \\
\hline 415 & 57.0 & 0.61 & 0.80 \\
\hline 915 & 55.0 & 0.34 & 0.95 \\
\hline 1500 & 54.0 & 0.26 & 1.18 \\
\hline 2000 & 53.0 & 0.25 & 1.45 \\
\hline
\end{tabular}

\section{Measurements}

Dielectric measurements are very fast and proceed through three steps. First the vector analyzer is calibrated. Then the calibration is checked using a reference material with a known dielectric constant $\varepsilon$ (e.g. distilled water, Fig. 3), and last but not least the reflection coefficient of the biological tissue is measured. The complex permittivity of biological tissue is evaluated using a personal computer and mathematical software (e.g. MatLab) to solve equation 6.

We measured two samples of biological tissue:

- sample A: a biological tissue of beef

- sample B: values measured on the author's arm

\section{Results}

A comparison of tabbed, modeled and calculated values:

\subsection{Comparison of relative permittivity}

Relative permittivity is a heavily frequency dependent quantity. Because of the decreassing ability of the particles, follow rapid changes of the electrical field, the relative permittivity decreases with increasing frequency.

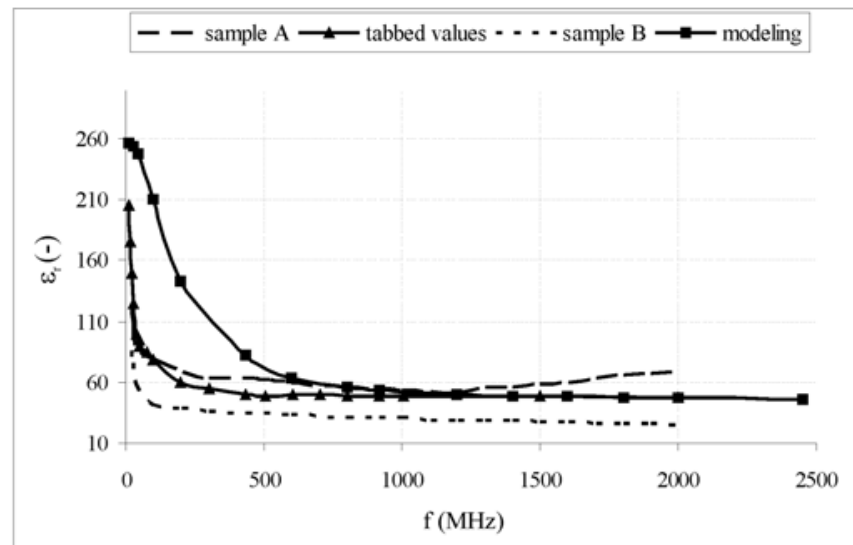

Fig. 8: Comparison of relative permittivity 
For muscle tissue, at low frequencies the real part of the relative permittivity has a value of about 200 , and with increasing frequency this goes down to a value of 50 .

\subsection{Comparison of the loss factor}

Loss factor $\operatorname{tg} \delta$ is also a frequency-dependent parameter. At low frequencies, there is a value of about 5 for muscle tissue $\operatorname{tg} \delta$, and with increasing frequency this value goes down to 0.35 .

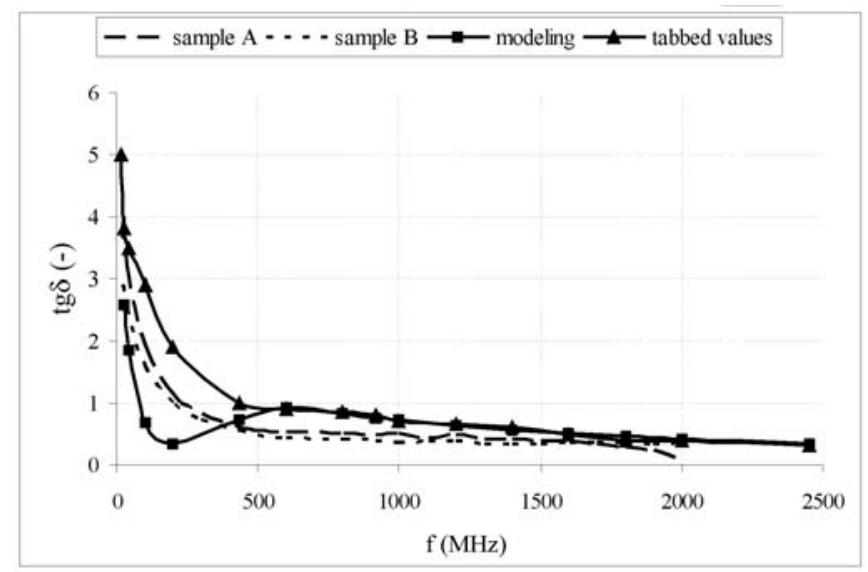

Fig. 9: Comparison of the loss factor

\section{Discussion}

The measurement method reported on here has an inconvenient calibration step, needing materials with known dielectric parameters. Total accuracy of the final determination of complex permittivity depends on accurate knowledge of this parameter. We very often use alcohol as a calibration material. Alcohols are hygroscopic liquids, so they are time-unsteady calibers. In addition their parameters are known from a table only for $100 \%$ virgin alcohols. Their use in calibration makes the measurements imprecise.

We will go on to study the relation between the dielectric constant of biological tissue and the reflection coefficient. By exploring the mathematical and physical model of the reflection coefficient measurement on the interface between biological tissue and the measurement sensor we can make the measurements more accurate. We will construct and compare the probes for various frequency bands. This will ultimately lead to total accuracy in determining of relative permittivity. The measurement will be thus more sensitive and therefore precise.

\section{Conclusion}

Measuring complex permittivity is a promising method for medical diagnostics and for preparing treatment with the use of an electromagnetic field. It is important to know the complex permittivity in the treatment area in order to design and match applicators for microwave thermotherapy. The reflection method on an open ended coaxial line is suitable for determining the dielectric parameters of biological tissue.

\section{Acknowledgments}

This work has been conducted at the Department of Electromagnetic Field at the Czech Technical University in Prague and has been supported by CTU grant no. CTU0607013.

\section{References}

[1] Vrba, J.: Medical Applications of Microwave Techniques. (in Czech) Prague: CTU in Prague Publishing house, 2003.

[2] Oppl, L.: Measurement of Dielectric Properties. (in Czech) Prague: Dissertation Thesis, Dept. of EM Field, CTU in Prague, 2001.

[3] Zajíček, R.: Dielectric Parameter Measurements of Biological Tissue. (in Czech) Prague: Diploma Thesis, Dept. of EM Field, CTU in Prague, 2005.

[4] Gabriel, S., Lau, R., W., Gabriel, C.: The Dielectric Properties of Biological Tissues: III. Parametric Models for the Dielectric Spectrum of Tissues. Phys. Med. Biol. Vol. 41 (1996), p. 2271-2293.

[5] Hazdra, P., Hudlička, M.: Finite Integration Technique, Modeling of Fields, Prague: IEEE Czechoslovakia Section, 2006, p. 41-48.

Ing. Radim Zajíček

e-mail: zajicer1@fel.cvut.cz

Prof. Ing. Jan Vrba, CSc.

Doc. Ing. Karel Novotný, CSc.

Department of Electromagnetic Field

http://www.elmag.org, http://www.hypertermie.cz

Czech Technical University in Prague

Faculty of Electrical Engineering

Technická 2

16627 Prague 6, Czech Republic 